\title{
Genome-wide identification of pathogenicity factors of the free-living amoeba Naegleria fowleri
}

Denise C Zysset-Burri ${ }^{1,2,3}$, Norbert Müller ${ }^{2}$, Christian Beuret ${ }^{1}$, Manfred Heller ${ }^{4}$, Nadia Schürch ${ }^{1}$, Bruno Gottstein ${ }^{2}$ and Matthias Wittwer ${ }^{1 *}$

\begin{abstract}
Background: The free-living amoeba Naegleria fowleri is the causative agent of the rapidly progressing and typically fatal primary amoebic meningoencephalitis (PAM) in humans. Despite the devastating nature of this disease, which results in $>97 \%$ mortality, knowledge of the pathogenic mechanisms of the amoeba is incomplete. This work presents a comparative proteomic approach based on an experimental model in which the pathogenic potential of $\mathrm{N}$. fowleri trophozoites is influenced by the compositions of different media.

Results: As a scaffold for proteomic analysis, we sequenced the genome and transcriptome of $N$. fowleri. Since the sequence similarity of the recently published genome of Naegleria gruberi was far lower than the close taxonomic relationship of these species would suggest, a de novo sequencing approach was chosen. After excluding cell regulatory mechanisms originating from different media compositions, we identified 22 proteins with a potential role in the pathogenesis of PAM. Functional annotation of these proteins revealed, that the membrane is the major location where the amoeba exerts its pathogenic potential, possibly involving actin-dependent processes such as intracellular trafficking via vesicles.

Conclusion: This study describes for the first time the $30 \mathrm{Mb}$-genome and the transcriptome sequence of $\mathrm{N}$. fowleri and provides the basis for the further definition of effective intervention strategies against the rare but highly fatal form of amoebic meningoencephalitis.
\end{abstract}

Keywords: Naegleria fowleri, Primary amoebic meningoencephalitis, Whole genome sequencing, RNA sequencing, Comparative proteomics, Pathogenicity factors

\section{Background}

Naegleria species are free-living amoebae found in soil and water throughout the world [1]. Although approximately 30 species have been recognized so far, Naegleria fowleri is the only human pathogen that causes primary amoebic meningoencephalitis (PAM) [2]. Infection occurs when water contaminated by $N$. fowleri enters the noses of swimmers and the amoebae reach the central nervous system through the olfactory nerve tract [3]. Several days after infection, patients suffer from severe inflammation of the brain and meninges, accompanied

\footnotetext{
* Correspondence: matthias.wittwer@babs.admin.ch

${ }^{1}$ Biology Division, Spiez Laboratory, Federal Office for Civil Protection, Austrasse, CH-3700 Spiez, Switzerland

Full list of author information is available at the end of the article
}

by headache, fever, vomiting, nausea and behavioral abnormalities. Because most infected individuals fail to be diagnosed rapidly, they die within one to two weeks after exposure to the infectious water source [3,4]. The drug of choice for treating PAM is the antifungal drug amphotericin B. However, no more than a dozen patients out of approximately 350 reported PAM cases have been treated successfully with amphotericin B, either alone or in combination with other drugs [5-7]. Hence, $N$. fowleri is very problematic due to the rapid onset and destructive nature of the disease as well as the lack of effective treatments, rather than the number of cases worldwide.

Knowledge of the genome of N. fowleri is needed to provide insights into the pathogenetic mechanisms of 
the amoeba as a basis for developing more effective therapies as well as more rapid diagnostic tools. Here, we present an approach consisting of whole-genome sequencing in combination with proteomic analysis for identifying potential pathogenicity factors in $N$. fowleri. The genome of its non-pathogenic relative Naegleria gruberi has recently been sequenced [8]. A comparative analysis of the genomes of $N$. gruberi and $N$. fowleri based on a $60-\mathrm{kb}$ nuclear segment showed less similarity between them than the present understanding of the phylogenetic relationships of Naegleria species would have led us to expect [9]. Therefore, the genome of $N$. gruberi is not suitable as a reference for genome assembly, and thus, a de novo sequencing approach had to be applied for determination of the complete genome sequence of $N$. fowleri. Furthermore, due to the substantial genetic differences observed, the application of a comparative genomic approach between pathogenic $N$. fowleri and non-pathogenic $N$. gruberi to define pathogenicity factors may be misleading. In the present work, we conducted an intra-species comparison of highly and weakly pathogenic $N$. fowleri trophozoites based on the model published by Burri et al. [10]. This model showed that $N$. fowleri trophozoites maintained in either Nelson's medium or PYNFH medium supplemented with liver hydrolysate (LH, PYNFH/LH medium) are highly pathogenic in mice and demonstrate rapid in vitro proliferation, whereas trophozoites cultured in PYNFH medium are weakly pathogenic with a slower growth. Although the pathogenicity cannot be explained by different cytotoxicity mechanisms or by the presence of membrane vesicles in this model, it enables to investigate the pathogenesis of $N$. fowleri under defined experimental conditions [10].

The evaluation of sequencing data is a computationally challenging task due to the volume of data involved and because of statistical interference in the algorithms used for elucidating the genomic organization of novel eukaryotic genomes. The identification of protein coding regions in de novo-sequenced eukaryotic genomes based solely on $a b$ initio computational algorithms is prone to specificity and sensitivity issues due to the lack of validated gene training sets. In this work, the obtained in silico gene-finding results were partially substantiated by experimental proteomic data. Furthermore, the search for potential pathogenicity factors was based on proteomic expression profiling of highly and weakly pathogenic $N$. fowleri, rather than, at least in this stage of research, less reliable transcriptomic data.

\section{Results}

\section{Genomic DNA sequencing}

As a pre-requisite for obtaining insight into the pathogenic mechanism of $N$. fowleri, the genome of the amoeba was sequenced using both Illumina HiSeq 2000 and Roche 454 GS FLX technology. The DNA isolated for wholegenome sequencing was composed of 2 times more plasmid DNA and 18 times less mitochondrial DNA than genomic DNA; thus, no substantial enrichment of either type of DNA was present in the starting material used for sequencing. In a first step, DNA was sequenced with the Illumina HiSeq 2000 platform, resulting in approximately 116 million $100-\mathrm{bp}$ paired-end reads with an insert size of $300 \mathrm{bp}$. The de novo assembly of these short 100 -bp reads was facilitated by 454 backbone sequencing, providing approximately 350,000 single reads with an average length of $378 \mathrm{bp}$. Finally, the assembly was improved with information from a mate-pair library composed of approximately 400 million Illumina reads with an insert size of $3 \mathrm{~kb}$. In total, over 500 million reads were de novo assembled into 1,124 scaffolds with an average coverage of 770x and an N50 of 136,406. The nuclear genome of $N$. fowleri has a size of 29,619,856 bp and is AT-rich, with a GC content of only $35.4 \%$ (Tables 1 and 2). The calculation of the genome size via flow cytometry indicated that the $N$. fowleri genome is approximately $66 \mathrm{Mb}$. Based on the 29,619,856-bp size of the de novo-assembled genome, the genome of $N$. fowleri is considered diploid.

\section{Transcriptome assembly and annotation}

To generate a database for protein identification via nano-liquid chromatography tandem mass spectrometry (nano-LC MS/MS), RNA was sequenced using the Illumina HiSeq 2000 platform, resulting in approximately 229 million 100-bp paired-end reads. Using Trinity and the program cd-hit for redundancy filtering, 17,252 open reading frames (ORFs) were predicted (Table 2 and Figure 1). These ORFs were then used as a database for the identification of proteins in the 2D gel spots and 1D gel slices (see below). Only $0.2 \%$ of the predicted ORFs failed to align to the draft genome.

The standalone BLASTp search against the RefSeqprotein database resulted in a significant hit for 16,021 of the 17,252 ORFs. To 7,820 of these 16,021 significant blast hits a Gene Ontology (GO) term could be assigned using the annotation pipeline Blast2GO (Figure 1).

Table 1 Summary of the $N$. fowleri genome

\begin{tabular}{ll}
\hline Parameter & Number \\
\hline Haploid genome size (bp) & $29,619,856$ \\
Sequence contigs (bp) & 4,339 \\
N50 of contigs (bp) & 17,724 \\
Sequence scaffolds & 1,124 \\
N50 of scaffolds (bp) & 136,406 \\
\hline
\end{tabular}


Table 2 Comparison of the $N$. fowleri with the $N$. gruberi genome

\begin{tabular}{lll}
\hline Parameter & N. fowleri & N. gruberi \\
\hline Haploid genome size (Mbp) & 29.62 & 40.96 \\
GC content (\%) & 35.4 & 33.1 \\
Open reading frames & 17,252 & 15,727 \\
Bidirectional best BLAST hit & & 13,495 \\
\hline
\end{tabular}

\section{Genome similarity}

As the genome of N. gruberi was sequenced in 2010 [8], there is an important question regarding the relationship of $N$. gruberi to other Naegleria species, especially to its pathogenic relative $N$. fowleri. To resolve the degree of relationships between $N$. gruberi and $N$. fowleri at the molecular level, we reconstructed a genome similarity network based on EST sequences (Figure 2). As additional groups for comparison, two other amoeba, Entamoeba histolytica and Acanthamoeba castellanii, belonging to class Amoebozoa, and Trypanosoma brucei and Trypanosoma cruzi, belonging to class Euglenozoa, which is the most closely related group to the Heterolobosea (including all Naegleria species), were used. In this network, $N$. fowleri and $N$. gruberi share substantially more gene families than $N$. fowleri shares with the other species assessed, thus positioning $N$. fowleri as the closest relative to $N$. gruberi. Regarding the other analyzed amoeba, $N$. fowleri is closer to $A$. castellanii than to E. histolytica.

In an additional approach, BLASTn searches suggested low similarity between the coding sequences of $N$. fowleri and N. gruberi, as only $32.1 \%$ of the 17,252 predicted ORFs aligned to the $N$. gruberi genome ( $>99.0 \%$ of the ORFs matched the de novo-assembled $N$. fowleri genome). Despite the low similarity on nucleotide level, $78.2 \%$ of the $N$. fowleri ORFs showed a BLASTp hit with $N$. gruberi genes (Table 2).

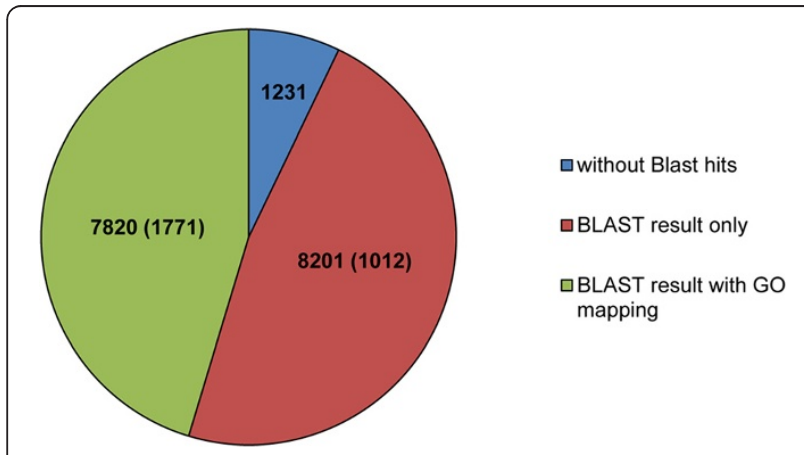

Figure 1 Overview of the $N$. fowleri genome annotation. From a total of 17,252 predicted open reading frames (ORFs), 1,231 remained without BLASTp hits, for 8,201 solely a BLASTp result was found and to 7,820 a Gene Ontology (GO) term could additionally assigned. The numbers in brackets indicate the number of ORFs that have been retrieved by proteomic analysis.

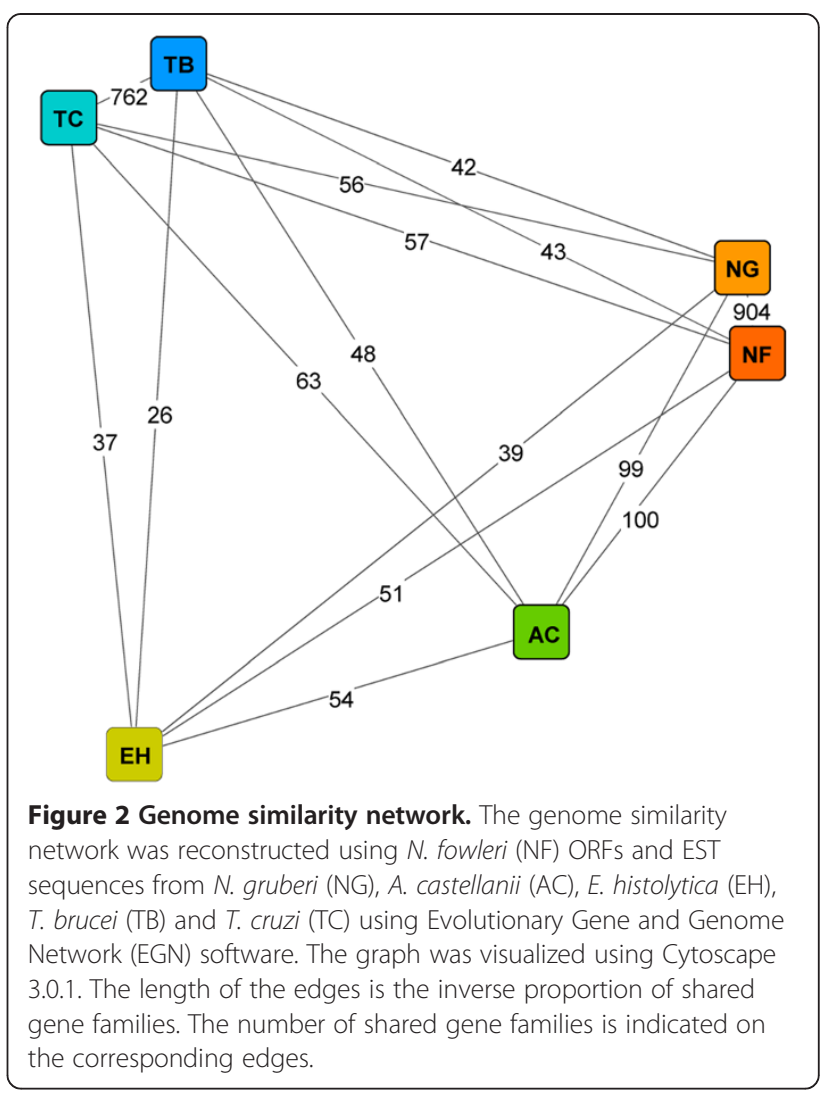

\section{Identification of potential pathogenicity factors}

The identification of pathogenicity factors in $N$. fowleri is an important step in revealing the mechanisms that are responsible for the destructive nature of PAM. Through comparative $2 \mathrm{D}$ gel electrophoresis, we obtained an overview of the proteomes of highly and weakly pathogenic $N$. fowleri (Figure 3). For comparative visual quantification of the obtained protein concentrations, the actinbinding protein cofilin, which was expressed at equivalent levels under the two conditions, was used as a control protein spot. As seen in Figure 3 and Table 3, heat shock protein 70 (hsp70) [11], actin 1 and 2 [11-18] as well as the membrane protein Mp2CL5 [19], all of which are known potential pathogenicity factors, were expressed at increased levels in highly pathogenic compared to weakly pathogenic trophozoites. This observation confirmed the potential of all four proteins to contribute to the pathogenicity of $N$. fowleri. Moreover, we identified cyclophilin as being strongly overexpressed in highly pathogenic trophozoites, suggesting that this protein is an additional potential pathogenicity factor. Conversely, another protein, the hsp20 domain-containing protein, was over-expressed in weakly pathogenic $N$. fowleri.

In a second step, by performing 1D gel electrophoresis in combination with nano-LC MS/MS (Figure 4), we detected a total of 2,166 proteins, 477 of which 

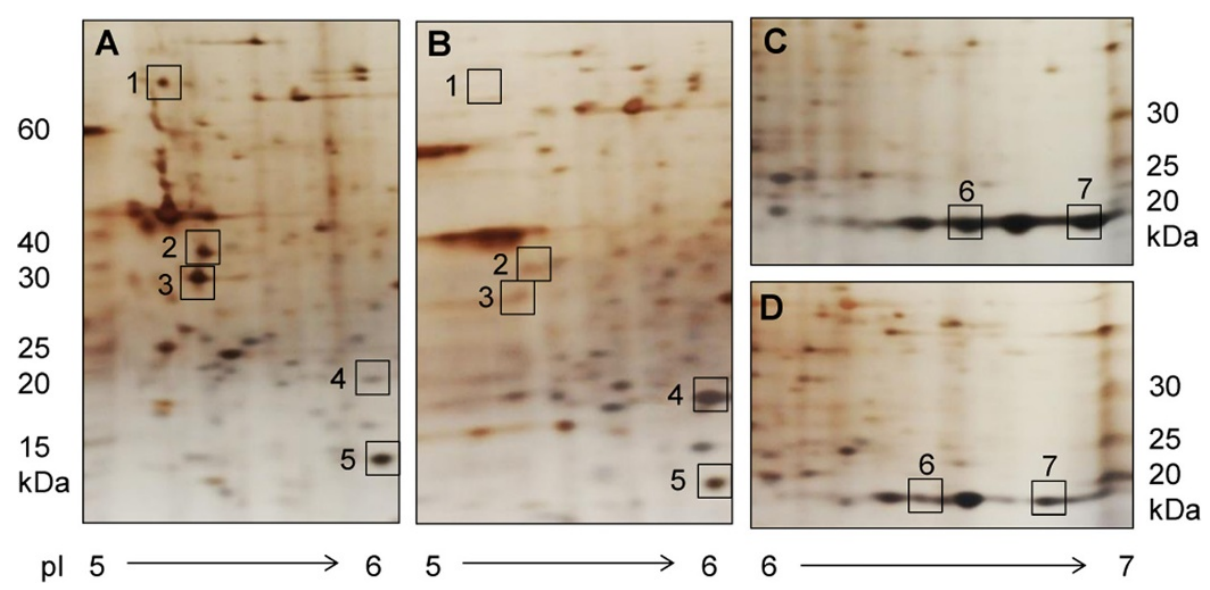

Figure 3 Comparative 2D gel electrophoresis of highly ( $A$ and $C$ ) and weakly (B and D) pathogenic $N$. fowleri. The proteomes of trophozoites were separated via 2D gel electrophoresis, and differentially expressed proteins (squares) were excised and identified via nano-liquid chromatography tandem mass spectrometry (nano-LC MS/MS). In the figure, only enlarged images from gel segments with differential spot patterns are shown. The numbers correspond to the identified proteins listed in Table 3. Spot 5 was used as a control, representing a protein (cofilin) with equivalent expression in highly and weakly pathogenic trophozoites.

were differentially expressed between weakly pathogenic trophozoites maintained in PYNFH medium and highly pathogenic trophozoites in PYNFH/LH medium (comparison group 1). Furthermore, 902 proteins were differentially expressed in weakly pathogenic trophozoites maintained in PYNFH medium and highly pathogenic trophozoites in Nelson's medium (comparison group 2). Approximately two-thirds of the proteins showing different expression levels could be annotated by ngKLAST (see Methods). Using the protein expression level in weakly pathogenic N. fowleri as a reference, we observed 175 up- and 174 down-regulated proteins in trophozoites grown in PYNFH/LH as well as 281 up- and 325 down-regulated proteins in trophozoites grown in Nelson's medium. Among these 950 differentially expressed and annotated proteins, only 99 were found to be up- or down-regulated in both highly pathogenic populations. Among these proteins, 43 were co-regulated in both highly pathogenic groups, while the remaining 56 were inversely regulated, i.e., up-regulated in one highly versus weakly pathogenic comparison group and down-regulated in the other comparison group. Among these 43 proteins that were co-regulated in both highly pathogenic N. fowleri populations, 22 components were up-regulated and were, thus, finally considered to represent potential pathogenicity factors.

Clustering of these newly identified potential pathogenicity factors from $N$. fowleri according to their cellular component GO affiliations suggested localization of the proteins to the cellular membrane, in vesicles and cell projections (Figure 5).

\section{Discussion}

Despite extensive research over the last several decades, the mechanisms accounting for the rapidly progressing and destructive nature of PAM are still unknown. Here, the $30-\mathrm{Mb}$ genome sequence of $N$. fowleri, including

Table 3 Proteins that were differentially expressed in highly and weakly pathogenic N. fowleri, as identified via 2D gel electrophoresis in combination with nano-LC MS/MS

\begin{tabular}{lllllll}
\hline Spot no & Accession & Protein description & Species & Mol. weight & Theoretical pl & Regulation \\
\hline 1 & AY684788 & Heat shock protein 70 (hsp70) & N. fowleri & 71408 Da & 5.14 & up \\
2 & M90311 & Actin 1 & N. fowleri & 41728 Da & 5.26 & up \\
3 & M90312 & Actin 2 & N. fowleri & 41153 Da & 5.23 & up \\
4 & XM_002673759 & Hsp20 domain containing protein & N. gruberi & 19836 Da & 6.20 & down \\
5 & XM_002669269 & Cofilin (control) & N. gruberi & 15384 Da & 5.90 & equal \\
6 & XM_002681214 & Cyclophilin & N. gruberi & 19333 Da & 6.29 & up \\
7 & AY049749 & Membrane protein Mp2CL5 & N. fowleri & 19932 Da & 6.82 & up \\
\hline
\end{tabular}

The molecular weight and theoretical pl values of the identified proteins were calculated according to the Compute $\mathrm{pl} / \mathrm{Mw}$ tool from the SIB Bioinformatics Resource Portal (http://web.expasy.org/compute_pi/). The results of visual quantification of the protein concentrations found in highly pathogenic compared to weakly pathogenic trophozoites are indicated in the last column (Regulation). 


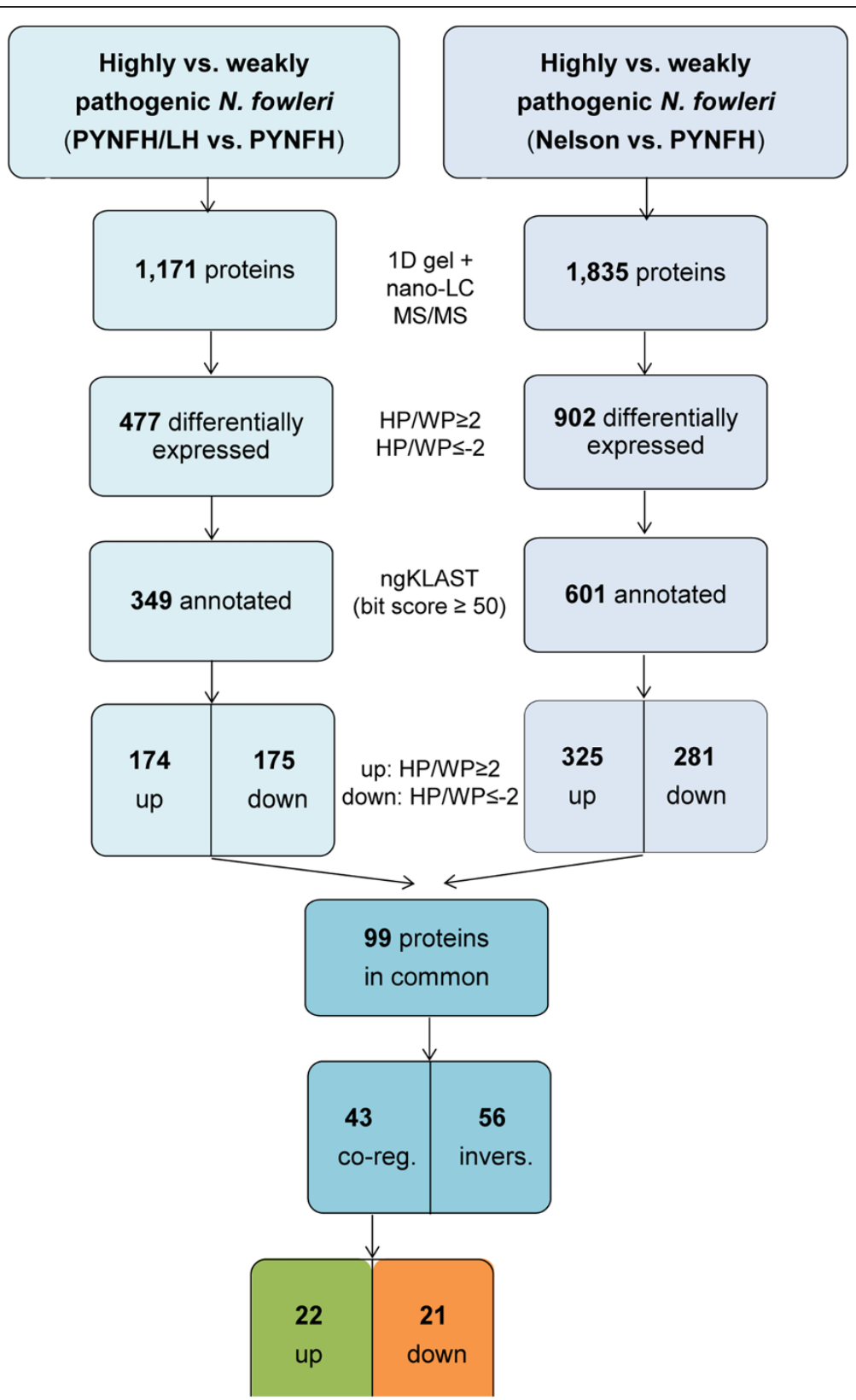

Figure 4 Workflow: Identification of potential pathogenicity factors in $\mathbf{N}$. fowleri. By performing $1 \mathrm{D}$ gel electrophoresis in combination with nano-liquid chromatography tandem mass spectrometry (nano-LC MS/MS), a total of 1,171 proteins were found in comparison group 1, i.e., highly pathogenic (HP) trophozoites in PYNFH/LH medium versus weakly pathogenic (WP) trophozoites in PYNFH medium; in comparison group 2, i.e., highly pathogenic trophozoites in Nelson's medium versus weakly pathogenic trophozoites in PYNFH medium, a total of 1,835 proteins were found. To identify proteins that were differentially expressed between highly and weakly pathogenic N. fowleri, a cut-off of a twofold change in expression (HP/WP $\geq 2$ for up-regulated (up) and HPMP $\leq-2$ for down-regulated (down) proteins) was chosen. Annotation by the program ngKLAST (www.korilog.com) with a bit score equal to or greater than 50 resulted in 349 annotated proteins in comparison group 1 and 601 annotated proteins in comparison group 2. Among the 99 proteins found to be up- or down-regulated in both comparison groups, 43 proteins were co-regulated (co-reg.), while 56 proteins were inversely (invers.) regulated, i.e., up-regulated in one and down-regulated in the other comparison group. Among the 43 co-regulated proteins, the 22 components that were up-regulated in the highly pathogenic trophozoites were considered to be potential pathogenicity factors in N. fowleri.

RNA sequencing data, is presented for the first time. Based on these data, we identified 22 novel potential pathogenicity factors in the amoeba using proteomic approaches. Moreover, 21 proteins were found to be down- regulated in highly pathogenic trophozoites. Most of this last group of proteins was composed of components of the mitochondrial respiratory chain as well as protein biosynthesis pathways (Table 4). Because they survive at 


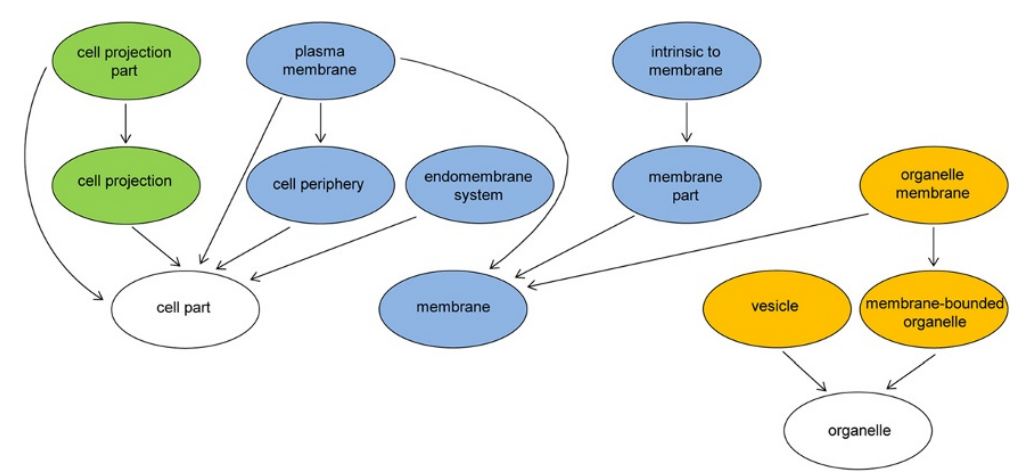

Figure 5 Cellular component analysis of potential pathogenicity factors from $\boldsymbol{N}$. fowleri. Gene Ontology (GO) graph generated from the cellular component ontology of the proteins, which were up-regulated in highly pathogenic trophozoites compared to weakly pathogenic trophozoites. The specificity of the GO terms increases from the bottom to the top of the graph with arrows indicating "being part of". The majority of the potential pathogenicity factors localize to the membrane of the cell (blue marked circles), whereas another group of proteins localized to either cell projections (green marked circles) or vesicles (orange marked circles).

the expense of their host, highly pathogenic $N$. fowleri trophozoites may require a smaller gene repertoire because they may benefit from the host's cells. In contrast, weakly pathogenic trophozoites are usually free living and therefore must possess all of the genes needed for essential metabolic processes, likely explaining the differences in the metabolism of the highly and weakly pathogenic $N$. fowleri.

From a phylogenetic point of view, the de novo-assembled $N$. fowleri genome may shed light on the extent of the taxonomic relationship between $N$. fowleri and its non-pathogenic relative $N$. gruberi, whose genome sequence was published in 2010 [8]. To the best of our knowledge, there is no reliable means, at least for eukaryotes, of translating whole-genome-based comparisons into taxonomic relationships. The general approach for assessing phylogenetic relationships is to pick a (set of) gene (s) as the basis for comparison. Using this approach, the level of divergence between $N$. fowleri and $N$. gruberi based on $18 \mathrm{~S}$ ribosomal DNA analysis has been estimated to be approximately similar to that between mammals and frogs [20]. In a study conducted by Herman et al. [9], a lack of collinearity between the $N$. fowleri and $N$. gruberi genomes was found through sequencing a 60-kb nuclear segment from $N$. fowleri and comparing it with corresponding sequences from $N$. gruberi. Furthermore, according to a typing system based on internal transcribed spacers and 5.8S rDNA sequences, there is strong evidence that Naegleria lovaniensis, and not $N$. gruberi, is the closest relative of $N$. fowleri [21]. According to the genome similarity network obtained by comparing $N$. fowleri with N. gruberi, A. castellanii, E. histolytica, T. brucei and T. cruzi based on EST sequences (Figure 2), we found that the extent of the relationship between $N$. fowleri and $N$. gruberi is comparable to that between T. brucei and T. cruzi. However, as only $32.1 \%$ of the assembled $N$. fowleri RNA transcripts aligned to the $N$. gruberi genome in BLASTn searches, we propose that there is low similarity between the coding sequences of $N$. gruberi and $N$. fowleri. In summary, all these findings reflect the intricate phylogeny of the protozoan taxonomy, and our data may add a further piece to this complex puzzle. In the context of our work, clarification of the phylogenetic relationships between Naegleria species is critical for choosing an appropriate search strategy for potential pathogenicity factors. Comparative analysis of genomic data from $N$. fowleri and $N$. gruberi, aimed at the identification of the pathogenic mechanisms of N. fowleri, has been discussed as a possible option in the field of Naegleria research. However, based on the findings described above, this experimental strategy is questionable because the substantial dissimilarities between the genomes of these species may lead to a high number of false positive candidates. In our opinion, the possibility of influencing the pathogenic potential of $N$. fowleri according to the composition of the culture medium is a more promising route for identifying relevant pathogenicity factors. While trophozoites maintained in PYNFH medium showed weak in vivo pathogenicity, trophozoites in Nelson's medium were highly pathogenic in mice. Furthermore, when the PYNFH medium was supplemented with LH, N. fowleri trophozoites also converted to the highly pathogenic phenotype [10]. Based on this pathogenicity model, we performed an intra-species comparison of $N$. fowleri using a genomic, transcriptomic and proteomic approach to identify the factors accounting for the pathogenic potential of the amoeba. To exclude proteomic differences caused by the different compositions of the media, we compared both of the highly pathogenic phenotypes (trophozoites in PYNFH/LH medium and trophozoites in Nelson's medium) with the weakly pathogenic trophozoites (cultivated in PYNFH medium). Among the 
Table 4 Differentially expressed proteins in highly and weakly pathogenic N. fowleri, as identified via 1D gel electrophoresis in combination with nano-LC MS/MS

\begin{tabular}{|c|c|c|c|c|}
\hline Accession Swissport & Hit Definition & Species & NP: HP/WP & PLP: HP/WP \\
\hline Q3SZP7 & Villin-1 & Bos taurus & 16.87 & 10.19 \\
\hline Q55585 & $\begin{array}{l}\text { Probable succinate-semialdehyde } \\
\text { dehydrogenase [NADP }(+)]\end{array}$ & $\begin{array}{l}\text { Synechocystis sp. PCC } 6803 \\
\text { substr. Kazusa }\end{array}$ & 9.09 & 11.63 \\
\hline Q4UB16 & Ras-related protein Rab-1 & Theileria annulata & 8.88 & 9.39 \\
\hline P08799 & Myosin II heavy chain & Dictyostelium discoideum & 8.03 & 3.86 \\
\hline Q4R550 & Cysteine-tRNA ligase, cytoplasmic & Macaca fascicularis & 7.35 & 4.79 \\
\hline B7MMS8 & $\begin{array}{l}\text { Gamma-aminobutyraldehyde } \\
\text { dehydrogenase }\end{array}$ & Escherichia coli 588 & 7.17 & 7.48 \\
\hline O75382 & Tripartite motif-containing protein 3 & Homo sapiens & 6.43 & 3.91 \\
\hline Q9V4N3 & Cytochrome b5; Short = CYTB5 & Drosophila melanogaster & 4.46 & 2.62 \\
\hline P27420 & Heat shock 70 kDa protein $C$ & Caenorhabditis elegans & 4.14 & 2.77 \\
\hline Q9N1T2 & $\begin{array}{l}\text { X-linked retinitis pigmentosa } \\
\text { GTPase regulator }\end{array}$ & Canis lupus familiaris & 4.12 & 4.97 \\
\hline D2VAA9 & $\begin{array}{l}\text { Methylthioribose-1-phosphate } \\
\text { isomerase }\end{array}$ & Naegleria gruberi & 3.91 & 2.90 \\
\hline Q25544 & $\begin{array}{l}265 \text { protease regulatory subunit } \\
8 \text { homolog }\end{array}$ & Naegleria fowleri & 3.88 & 2.50 \\
\hline P54772 & Histidine decarboxylase & Solanum lycopersicum & 2.91 & 8.22 \\
\hline P10733 & Severin & Dictyostelium discoideum & 2.88 & 2.73 \\
\hline Q1HPW4 & $\begin{array}{l}\text { Eukaryotic translation initiation } \\
\text { factor } 3 \text { subunit I }\end{array}$ & Bombyx mori & 2.85 & 2.23 \\
\hline Q54BW4 & Circularly permutated Ras protein 2 & Dictyostelium discoideum & 2.46 & 3.79 \\
\hline Q12965 & Unconventional myosin-le & Homo sapiens & 2.30 & 3.56 \\
\hline P34552 & $\begin{array}{l}\text { Apoptosis-linked gene } 2 \text {-interacting } \\
\text { protein X } 1\end{array}$ & Caenorhabditis elegans & 2.30 & 2.21 \\
\hline Q54K50 & Phospholipase D Y & Dictyostelium discoideum & 2.22 & 2.19 \\
\hline Q5TJ55 & Formin-D & Dictyostelium discoideum & 2.18 & 8.77 \\
\hline Q1ZXF7 & GDP-mannose 4,6 dehydratase & Dictyostelium discoideum & 2.15 & 2.44 \\
\hline Q8IV36 & Protein HID1 & Homo sapiens & 2.07 & 2.71 \\
\hline P13629 & $\begin{array}{l}\text { Periplasmic [Fe] hydrogenase large } \\
\text { subunit }\end{array}$ & $\begin{array}{l}\text { Desulfovibrio oxamicus } \\
\text { (strain Monticello) }\end{array}$ & -2.14 & -2.86 \\
\hline P08964 & Myosin-1 & $\begin{array}{l}\text { Saccharomyces cerevisiae } \\
\text { S288c }\end{array}$ & -2.25 & -5.91 \\
\hline P51824 & ADP-ribosylation factor 1 & Solanum tuberosum & -2.38 & -6.44 \\
\hline A7HBL7 & Elongation factor Tu & Anaeromyxobacter sp. Fw109-5 & -2.46 & -2.42 \\
\hline Q9CR62 & $\begin{array}{l}\text { Mitochondrial 2-oxoglutarate/malate } \\
\text { carrier protein }\end{array}$ & Mus musculus & -2.56 & -3.14 \\
\hline Q1ZXF1 & $\begin{array}{l}\text { Probable enoyl-CoA hydratase, } \\
\text { mitochondrial }\end{array}$ & Dictyostelium discoideum & -2.69 & -4.44 \\
\hline F4P6T0 & Ubiquinol oxidase, mitochondrial & $\begin{array}{l}\text { Batrachochytrium dendrobatidis } \\
\text { JAM81 }\end{array}$ & -2.97 & -5.61 \\
\hline P77735 & Uncharacterized oxidoreductase YajO & Escherichia coli K-12 & -3.06 & -2.15 \\
\hline Q889U1 & Single-stranded DNA-binding protein & $\begin{array}{l}\text { Pseudomonas syringae pv. } \\
\text { tomato str. DC3000 }\end{array}$ & -3.19 & -3.39 \\
\hline P48375 & 12 kDa FK506-binding protein & Drosophila melanogaster & -3.55 & -2.95 \\
\hline Q2G8K9 & Elongation factor Ts & $\begin{array}{l}\text { Novosphingobium aromaticivorans } \\
\text { DSM } 12444\end{array}$ & -3.56 & -2.27 \\
\hline Q756S4 & Mitochondrial import inner membrane & Neurospora crassa OR74A & -3.67 & -2.80 \\
\hline
\end{tabular}




\begin{tabular}{|c|c|c|c|c|}
\hline P54168 & Uncharacterized protein YpgQ & Bacillus subtilis subsp. subtilis str. 168 & -3.9 & -3.05 \\
\hline О42899 & Protein scol & Schizosaccharomyces pombe 972 h- & -3.91 & -7.12 \\
\hline Q97FZ9 & Rubrerythrin-1 & Clostridium acetobutylicum ATCC 824 & -4.94 & -2.06 \\
\hline Q9P7M0 & ABC1 family protein C21C3.03, mitochondrial & Schizosaccharomyces pombe 972 h- & -5.73 & -2.62 \\
\hline P42730 & Chaperone protein CIpB1 & Arabidopsis thaliana & -6.29 & -4.14 \\
\hline P54872 & Hydroxymethylglutaryl-CoA synthase A & Dictyostelium discoideum & -6.3 & -3.2 \\
\hline Q60597 & 2-oxoglutarate dehydrogenase, mitochondrial & Mus musculus & -8.13 & -2.87 \\
\hline P42125 & Enoyl-CoA delta isomerase 1, mitochondrial & Mus musculus & -8.93 & -2.44 \\
\hline O67589 & Aspartate-tRNA ligase & Aquifex aeolicus VF5 & -20.48 & -11.26 \\
\hline
\end{tabular}

The proteins are sorted according to their decreasing level of up- or down-regulation in reference to weakly pathogenic $N$. fowleri. The regulation levels are given in columns 4 (NP: HP/WP: highly pathogenic trophozoites in Nelson's medium versus weakly pathogenic trophozoites in PYNFH medium) and 5 (PLP: HP/WP: highly pathogenic trophozoites in PYNFH/LH medium versus weakly pathogenic trophozoites in PYNFH medium). The 22 up-regulated proteins (HP/WP > 2) were considered to be potential pathogenicity factors of $N$. fowleri.

950 initially identified ORFs showing one or more peptide matches in the mass spectrometric analysis, only 22 proteins were up-regulated in both comparison groups and were therefore considered potential pathogenicity factors.

The pathogenicity of an organism is a complex process and is proposed to result from the interactions of many components, rather than the action of one essential factor. Thus, we clustered the 22 potential pathogenicity factors identified according to their cellular components to determine the compartment with the highest pathogenic activity (Figure 5).

\section{(Trans-) membrane domain}

Based on the GO assignment of the proteins to their cellular locations, the membrane was proposed to be one of the main foci where pathogenic activity occurs. Because adherence of $N$. fowleri to its host cells is a crucial step in inducing a successful infection [22], the membrane (i.e., transmembrane proteins) may play an important role in the pathogenesis of PAM. Based on a previous investigation, a fibronectin-binding protein essential for the interaction of trophozoites with extracellular matrix glycoproteins was identified, suggesting that $N$. fowleri harbors a membrane protein related to the human integrin-like receptor [23]. Furthermore, several studies have shown that $N$. fowleri lyses a wide variety of mammalian target cells in vitro through contact-dependent mechanisms [24-26]. This is a further indication of the presence of surface proteins with an essential role in the lytic activity of trophozoites. Lowrey and McLaughlin identified a membrane-associated protein with cytolytic activity against mammalian cells [27]. Another membrane protein, Mp2CL5, was isolated from pathogenic $N$. fowleri and was not found in non-pathogenic Naegleria species, suggesting Mp2CL5 as a potential pathogenicity factor [19]. Because Mp2CL was expressed at higher levels in highly pathogenic compared to weakly pathogenic trophozoites, as accessed via comparative $2 \mathrm{D}$ gel electrophoresis (Figure 3, Table 3), our analysis confirmed the potential involvement of this membrane protein in the pathogenesis of $N$. fowleri. Thus, we consider Mp2CL5 an important candidate for further examination of its role in the pathogenesis of PAM.

In another study, different membrane-bound glycoproteins involved in resistance to complement-mediated damage were described [28]. Moreover, Fritzinger et al. demonstrated the presence of an immunogenic surface protein in $N$. fowleri that was reactive with antibodies to human CD59. Because this CD59-like protein binds complement component C9, it may play a role in resistance to complement lysis. Additionally, it has been shown that the CD59-like protein is shed on membrane vesicles [29]. Generally, N. fowleri undergoes membrane vesiculation to remove membranedeposited $C$ proteins, thereby protecting the amoeba from complement damage [30]. In the present study, we also identified vesicle trafficking as a potential pathogenicity mechanism (see the following section).

\section{Vesicular trafficking}

As mentioned above, $N$. fowleri undergoes membrane vesiculation as a mechanism for resisting complement damage [30]. Because various proteins identified as likely to be involved in the pathogenic mechanisms of $N$. fowleri are stored and ultimately shed in membrane vesicles, vesicular trafficking may play an important role in the pathogenesis of PAM. The CD59-like protein mentioned above is shed in vesicles [29]. Furthermore, the two pore-forming glycoproteins, naegleriapore A and B, are stored in intracellular granular vesicles. As naegleriapore $\mathrm{A}$ and $\mathrm{B}$ exert cytotoxicity in the form of membranepermeabilizing activity towards prokaryotic as well as eukaryotic cells, they are proposed to be involved in the pathogenesis of PAM [31]. The vesicular storage of the CD59-like protein, naegleriapores A and B and likely also other potential $N$. fowleri pathogenicity factors may 
present a means of self-protection from the cytotoxic activity of these factors. Therefore, intracellular vesicles may function as part of a pathogenicity machinery via storing and ultimately secreting proteins that are able to destroy target cells.

Previous studies conducted in our lab have demonstrated the localization of membrane vesicles on highly pathogenic trophozoites maintained in Nelson's medium, but not on weakly pathogenic trophozoites. However, because no vesicle formation was observed in those trophozoites in PYNFH/LH medium, which were also found to be highly pathogenic, the presence of membrane vesicles could not be related to the in vivo pathogenicity [10]. Conversely, based on a combination of findings, the vesicular trafficking system per se was characterized as a cellular compartment with potential pathogenic activity (Figure 5). In particular, it was found that apoptosislinked gene-2-interacting protein X1 (AIP1), which has now been identified as a potential $N$. fowleri pathogenicity factor (Table 4), is a key regulator of endosomal sorting [32]. The endosomal system accomplishes the intracellular transport of cellular material between organelles such as the Golgi apparatus as well as from organelles to the membrane and vice versa via vesicles. Yu et al. suggested that the Golgi-localized transmembrane protein HID-1, which is up-regulated in highly pathogenic N. fowleri (Table 4), may be involved in vesicular exocytosis by preventing the mis-sorting of peptides to lysosomes for degradation [33,34]. Thus, both AIP1 and HID-1 are interesting candidate $N$. fowleri pathogenicity factors, potentially acting to regulate vesicular trafficking in the amoeba.

In E. histolytica it has been shown that, in addition to the storage and secretion of cytolytic molecules (such as amoebapores and cytolytic cysteine proteases), vesicles are implicated in phagocytosis [35-37]. The Rab GTPase EhRabB, which is localized in cytoplasmic vesicles, is involved in the phagocytosis of E. histolytica [37,38]. Rho family GTPases, including Rab proteins such as EhRabB, regulate the cytoskeleton and associated pathogenic processes such as phagocytosis, which in turn, is controlled by vesicular trafficking [39]. Although this topic requires further investigation, the Ras-related protein Rab-1, which was up-regulated in highly pathogenic compared to weakly pathogenic $N$. fowleri in our analysis (Table 4), may be involved in vesicular trafficking and, thus, in the phagocytosis of target cells.

Taking these findings together, vesicular trafficking may be an important step in the pathogenesis of $N$. fowleri infection, as potential pathogenicity factors in the amoeba, including the CD59-like protein and naegleriapores $\mathrm{A}$ and $B$, are stored in vesicles. This possibility is further supported by our analysis showing that vesicular trafficking is regulated by proteins identified as potential pathogenicity factors in N. fowleri, such as AIP1, Rab1 and HID-1.

The formation of vesicles via membrane budding involves re-organization of the cytoskeleton, mainly depending on the turnover of actin filaments [30], which is discussed as a potential factor in the pathogenicity of $N$. fowleri in the next section.

\section{Cell projection}

Cell projection was identified as a process that is likely involved in the pathogenesis of PAM (Figure 5). Naegleria trophozoites exhibit amoebastomes, or food-cups, which are pseudopodial projections $[24,40]$. These amoebastomes are involved in the attachment of amoebae to substrates as well as in the ingestion of bacteria, yeast cells and cellular debris via phagocytic processes [24,26,40,41]. Phagocytosis is dependent on the dynamic turnover of the cytoskeletal protein actin. Because actin is localized around food cups and has been shown to have the capacity to modulate in vitro cytotoxicity in different target cells, it is frequently discussed as a potential pathogenicity factor in $N$. fowleri [11-16,18]. Furthermore, the effects of immunization with either a DNA vaccine or a lentiviral vector expressing the $n f a 1$ gene $(N$. fowleri actin 1$)$ were investigated in mice infected with PAM $[42,43]$. In the present study, actin 1 and actin 2 were found to be upregulated in highly pathogenic trophozoites in 2D gels (Figure 3, Table 3), confirming the potential role of actin in the pathogenic mechanisms of $N$. fowleri. Another protein showing specific localization around phagocytic food cups that plays a role in cytotoxicity as well as in proliferation of $N$. fowleri is heat shock protein 70 (hsp70) [44]. The potential involvement of hsp70 in the pathogenic mechanisms of the amoeba was confirmed by our analysis detecting the up-regulation of this protein in highly pathogenic trophozoites (Figure 3, Tables 3 and 4).

The structural function of the actin cytoskeleton is essential in eukaryotic cells. Actin filaments participate in diverse cellular processes, such as adhesion [45], motility [46] and phagocytosis. The role of actin during phagocytosis has also been reported in other parasites, including E. histolytica [47] and A. castellanii [48]. Because actin plays a dynamic and structural role in many essential mechanisms in cells, its turnover must be strictly regulated by actin-binding proteins (Figure 6). Formins, such as formin $\mathrm{D}$, which we identified as a potential pathogenicity factor in $N$. fowleri (Table 4), are a crucial class of proteins that regulate the formation of actin filaments. Formins promote the polymerization of monomeric G-actin into filamentous F-actin as well as actin nucleation (Figure 6), ultimately resulting in actin filament assembly through a processive capping mechanism $[49,50]$. Another actin-modifying protein with a potential role in the pathogenesis of PAM is severin (Table 4). 


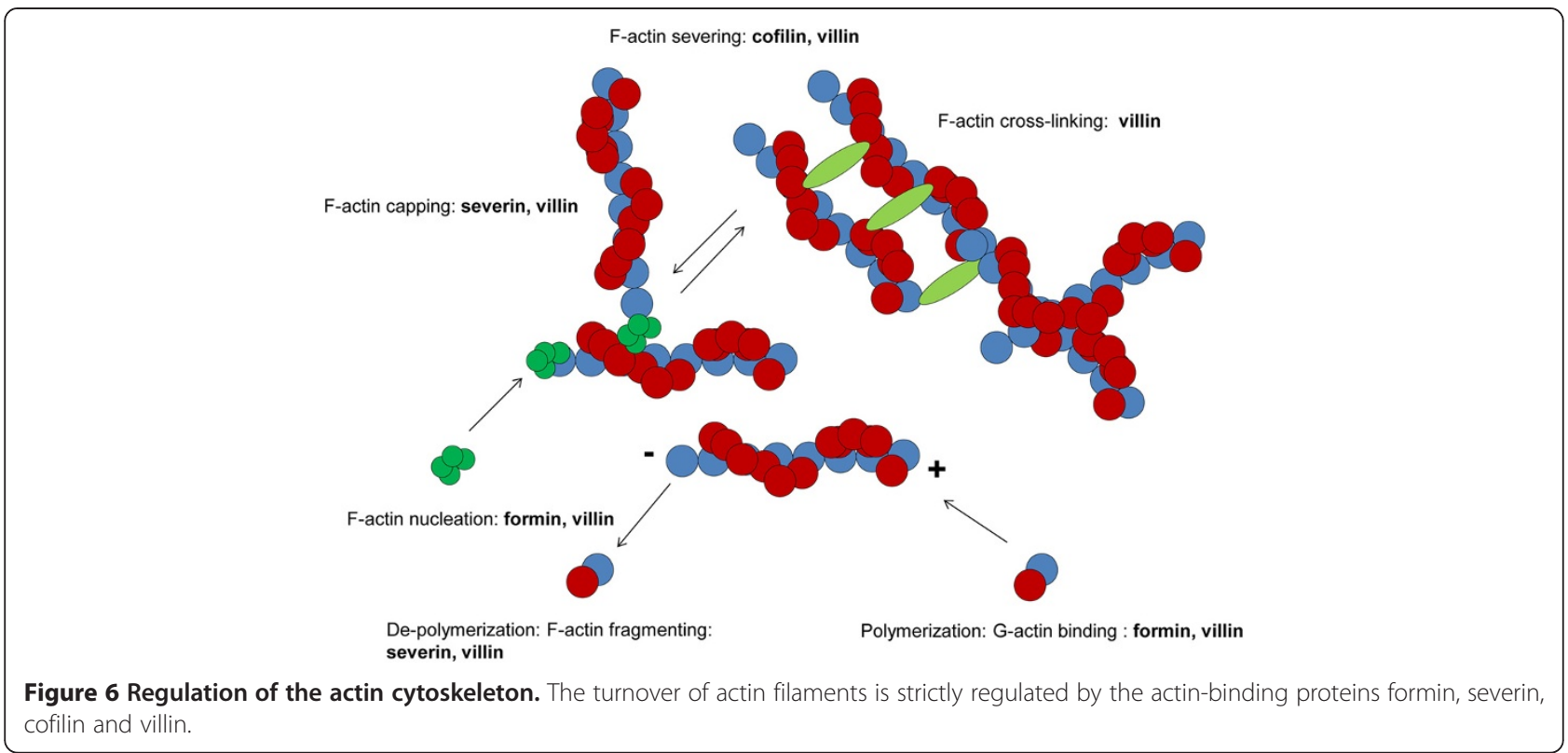

Severin belongs to the class of actin-fragmenting and -capping proteins (Figure 6) [51]. F-actin fragmentation is required for rapid re-arrangements of the filamentous actin cytoskeleton during cellular processes such as phagocytosis. Cofilin, which showed equal expression levels in weakly and highly pathogenic trophozoites in 2D gels (Figure 3, Table 3), is an actin filament-severing protein that creates free barbed ends that are available for de-polymerization or polymerization (Figure 6) [52,53]. Finally, villin is a multi-functional actin cytoskeletonregulating protein that is able to perform all of the actin-modifying functions discussed above, carrying out actin nucleation and polymerization as formin does; actin capping and fragmentation as severin does; actin severing as cofilin does; and actin bundling via cross-linking actin filaments (Figure 6) [54]. In our analysis, villin-1 was the protein found to be up-regulated at the highest level in highly pathogenic $N$. fowleri compared to weakly pathogenic N. fowleri (Table 4). Though the exact role of retinitis pigmentosa GTPase regulator (RPGR), which was also identified as a potential pathogenicity factor in $N$. fowleri (Table 4), is unknown, Gakovic et al. proposed the involvement of RPGR in the regulation of F-actin [55]. Although this possibility requires further investigation, formin D, severin, villin-1 and RPGR may be involved in actin-dependent pathogenic processes such as phagocytosis. Because of the versatile role of villin-1 in regulating the actin cytoskeleton and the fact that it showed the highest level of up-regulation in highly pathogenic trophozoites, villin-1 is the most promising candidate for further investigations to elucidate the molecular mechanisms involved in the pathogenesis of PAM.
As noted above, phagocytosis is an actin-dependent process. Dianokova et al. [56] showed that the actinbinding myosins are concentrated around phagocytic cups in macrophages. Based on the notion that these phagocytic cups are similar to amoebastomes, myosin may be involved in phagocytic processes in the amoeba. We identified myosin II heavy chain as well as myosin Ie as potential pathogenicity factors in N. fowleri (Table 4). In macrophages, myosin II is required for the contractile activity of phagocytic cups [57], whereas class I myosins have been proposed to act at the membrane-actin interface to support endocytosis and exocytosis via vesicular trafficking [58]. Thus, further experiments are required to investigate the putative localization of myosin at the site of amoebastomes and to examine its role in the phagocytosis of target cells.

\section{Conclusions}

Using genomic, transcriptomic and proteomic approaches, we identified 22 proteins that potentially act as pathogenicity factors in the deadly amoeba $N$. fowleri. The membrane was identified as a key location where pathogenic processes may occur, and these processes most likely involve actin-dependent vesicular trafficking mechanisms. This study will be the basis for our future application of reverse genetic approaches to demonstrate the role of the identified candidate proteins in the pathogenesis of PAM.

\section{Methods}

\section{In vitro cultivation of $\boldsymbol{N}$. fowleri}

Weakly pathogenic $N$. fowleri trophozoites (ATCC 30863) were cultivated at $37^{\circ} \mathrm{C}$ in $5 \mathrm{ml}$ of buffered PYNFH medium containing $1 \%(\mathrm{w} / \mathrm{v})$ Bacto $^{\mathrm{mw}}$ Peptone (BD Biosciences, 
Allschwil, Switzerland), 1\% (w/v) yeast extract (BD Biosciences), 0.1\% (w/v) yeast ribonucleic acid (Sigma, Buchs, Switzerland), $15 \mathrm{mg}$ folic acid (Sigma) $\mathrm{l}^{-1}$ and $1 \mathrm{mg}$ hemin (Sigma) $\mathrm{l}^{-1}$, supplemented with $10 \%(\mathrm{v} / \mathrm{v})$ fetal

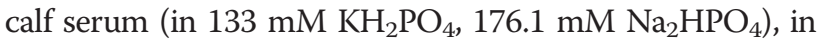
Nunclon $^{\mathrm{TM}} \Delta$ Surface tubes (Fisher Scientific, Allschwil, Switzerland) from frozen stocks. To generate highly pathogenic $N$. fowleri, trophozoites were transferred either to Nelson's medium containing $0.1 \%$ (w/v) LH (Sigma) and $0.1 \%(w / v)$ D-(+)-glucose (Sigma), supplemented with $10 \%$ $(\mathrm{v} / \mathrm{v})$ fetal calf serum in Page's amoeba saline $(2 \mathrm{mM} \mathrm{NaCl}$, $16 \mu \mathrm{M} \mathrm{MgSO} 4,27.2 \mu \mathrm{M} \mathrm{CaCl}_{2}, 1 \mathrm{mM} \mathrm{Na} \mathrm{HPO}_{4}, 1 \mathrm{mM}$ $\mathrm{KH}_{2} \mathrm{PO}_{4}$ ), or to PYNFH medium supplemented with $0.1 \%$ $(\mathrm{w} / \mathrm{v}) \mathrm{LH}[10]$.

\section{Genomic DNA sequencing}

\section{DNA isolation and library preparation}

DNA was extracted from $10^{8} \mathrm{~N}$. fowleri trophozoites cultivated in Nelson's medium using the DNeasy Blood and Tissue Kit (Qiagen, Basel, Switzerland) according to the manufacturer's protocol. To obtain RNA-free DNA, RNA digestion was performed using $4 \mu$ of RNase A (Qiagen). The DNA was eluted with $100 \mu \mathrm{l}$ of $10 \mathrm{mM}$ TrisHCl, $\mathrm{pH} 8.5$, pre-heated to $70^{\circ} \mathrm{C}$. The DNA quality was visualized on $0.8 \%$ agarose gels, and quantification was performed using a NanoDrop ${ }^{\varpi}$ device. Three micrograms of high-molecular weight DNA was sent to Fasteris (Planles-Quates, Switzerland) for paired-end sequencing, with an insert size of $300 \mathrm{bp}$, using the Illumina HiSeq 2000 platform, while $20 \mu \mathrm{g}$ of DNA was sent to GATC Biotech (Constance, Germany) for preparation of a 3-kb mate-pair library using Illumina technologies and for Roche 454 GS FLX sequencing.

The NGS reads have been deposited in DDBJ/EMBL/ GenBank under accession SRX523949 (Illumina HiSeq 2000 reads) and SRX547942 (Roche 454 GS FLX reads).

\section{PCR analysis of the contents of ribosomal and mitochondrial DNA relative to the genomic DNA}

In addition to its nuclear genome, $N$. fowleri has multiple copies of an extrachromosomal plasmid encoding ribosomal DNA $[59,60]$ and a 50-kb mitochondrial genome [9]. To avoid bias in the contents of ribosomal and mitochondrial DNA relative to genomic DNA, DNA extracted for whole-genome sequencing was subjected to PCR analysis specifically targeting $18 \mathrm{~S}$ rDNA, mitochondrial DNA and a glutathione $S$ transferase III homolog (EMBL:U43126) in the genome. The primers used for PCR are listened in the Additional file 1.

Genome size and ploidy level estimation via flow cytometry Flow cytometry is a method that is widely used to measure genome sizes in plants [61]. We estimated the genome size and level of ploidy of $N$. fowleri based on the known genome sizes of Giardia lamblia and Trichomonas vaginalis. Pellets of approximately $10^{7} \mathrm{~N}$. fowleri, G. lamblia and T. vaginalis were diluted in PBS to a concentration of $10^{5}$ trophozoites $/ \mathrm{ml}$. To stain the nuclei, $20 \mu \mathrm{l}$ of SYBR Green I (Invitrogen, Lucerne, Switzerland), which intercalates into the DNA [62], was added to the samples, followed by incubation for 20 minutes in the dark. Flow cytometric detection was performed with a Partec CyFlow $^{\ominus}$ SL flow cytometer (Partec $\mathrm{GmbH}$, Münster, Germany) equipped with a 488-nm blue solid-state laser operating at $20 \mathrm{~mW}$. The trigger was set to green fluorescence. The flow speed rate was $3 \mu \mathrm{l} /$ second, implying a counting rate of less than $10^{3}$ events/second. The results were acquired using Partec FlowMax software, v2.4d. The calculation of the genome size of $N$. fowleri was based on the known genome sizes of the reference species (e.g., $48 \mathrm{Mb}$ for G. lamblia [63] and $177 \mathrm{Mb}$ for $T$. vaginalis [64]) and the relative green fluorescence in the co-prepared samples [65].

\section{De novo assembly}

Because no reference genome exists for $N$. fowleri, the sequencing reads were assembled de novo using CLC 4.7.1. Raw reads were trimmed for removal of low-quality sequences (with a limit of 0.05) and for ambiguous nucleotides. The Roche 454 read ends were additionally screened and trimmed for 454 adapter sequences. Genome assembly was performed with the CLC de novo sequencing tool using the default parameters, with a minimum contig length of $800 \mathrm{bp}$ and with scaffolding. CLC assembly is a two-step process based on the De Bruijn graph algorithm. First, contig sequences are built based on information included in the read sequences, such as paired-end information. In a second step, to show the coverage levels, all reads are mapped back using the contig sequences obtained as a reference.

The results of this whole-genome shotgun project have been deposited in DDBJ/EMBL/GenBank under accession AWXF00000000 and in the eukaryotic pathogen database EuPathDB (http://eupathdb.org/eupathdb/). The version described in this paper is version AWXF01000000.

\section{RNA sequencing}

\section{RNA isolation and library preparation}

RNA was extracted from $10^{7} \mathrm{~N}$. fowleri trophozoites cultivated in Nelson's medium using the EZ1 RNA Universal Tissue Kit (Qiagen) and the EZ1 BioRobot (Qiagen). Trophozoites were resuspended in $750 \mu \mathrm{l}$ of QIAzol lysis reagent (Qiagen), followed by disruption and homogenization by operating a TissueLyser at $25 \mathrm{~Hz}$ for $3 \mathrm{~min}$. After incubation for $5 \mathrm{~min}$ at room temperature, $150 \mu \mathrm{l}$ chloroform (Grogg, Stettlen, Switzerland) was added to the homogenized samples. The mixture was then centrifuged for $15 \mathrm{~min}$ at $12,000 \mathrm{~g}$ at $4^{\circ} \mathrm{C}$, and the upper aqueous phase 
was used as the starting material for RNA isolation with the EZ1 BioRobot, according to manufacturer's protocol. Quantification and examination of the total RNA integrity was performed with the Agilent 2100 Bioanalyzer system. Four micrograms of high-quality RNA was sent to the Next Generation Sequencing Platform of the University of Bern for paired-end sequencing by the Illumina HiSeq 2000 device.

The reads from RNA sequencing have been deposited in DDBJ/EMBL/GenBank under accession SRX553040.

\section{De novo assembly and ORF prediction}

To obtain high-quality transcriptome sequence data, raw reads were trimmed via the removal of low-quality sequences (with a limit of 0.05) and based on ambiguous nucleotides. The trimmed reads were then de novo-assembled into transcripts with Trinity, a three-module software pipeline specifically developed for de novo transcriptome assembly [66]. Trinity generates contigs, clusters the contigs into individual groups, with each representing the full transcriptional complexity of a given gene, and then constructs a De Brujin graph for each contig group. To identify ORFs, protein-coding regions were extracted from Trinity transcripts by a downstream application of the program. High redundant ORFs were filtered over a 95\% identity threshold using the program cd-hit (http:// weizhong-lab.ucsd.edu/cd-hit/). The resulting ORFs were used as a database for protein identification via nano-LC MS/MS (see below). To assess the accuracy of the assembled transcripts, each transcript was aligned to our genomic data using the CLC Mapping tool with the default parameters.

\section{Genome similarity of N. fowleri and N. gruberi}

To compare the genetic diversity of the genome of $N$. fowleri with its non-pathogenic relative $N$. gruberi and with the more distantly related species $A$. castellanii, E. histolytica, T. brucei and T. cruzi, Evolutionary Gene and Genome Network (EGN) software was used [67]. EGN generates genome networks from molecular datasets by comparing sequences via BLAST homology searches. As input files for comparison with our genefinding data, EST sequences from N. gruberi, A. castellanii, E. histolytica, T. brucei and T. cruzi were downloaded from the National Center for Biotechnology Information website (NCBI, http://www.ncbi.nlm.nih.gov/). The genome network was generated at an e-value cutoff of 3 and a $20 \%$ identity threshold. The EGN output file was imported into Cytoscape 3.0.1 [68] to visualize the genome network as a graph, with nodes representing the organisms and edges representing the similarity between two nodes (Figure 2). The length of an edge is represented as the inverse proportion of shared gene families.
Moreover, the 17,252 predicted ORFs from N. fowleri were queried against the genome of $N$. gruberi (NCBI: ACER00000000.1) as well as against the de novo-sequenced genome of $N$. fowleri using BLASTn [69]. The applied parameters were as follows: match $=2$, mismatch $=-3$, gap costs for existence $=5$ and for extension $=2$. The minimal hit length was set to 100 nucleotides.

For standalone BLASTp protein comparison against the RefSeq database, default parameters were applied. The resulting XML file was then used for functional annotation by the CLC plugin Blast2GO.

\section{Proteomics}

\section{D gel electrophoresis}

To identify potential pathogenicity factors in N. fowleri, the proteomes of weakly and highly pathogenic trophozoites were separated via 2D gel electrophoresis, and differing protein spots were analyzed through nano-LC MS/ MS. Pellets of $10^{7}$ trophozoites were washed 3 times in PBS, and $10 \mu \mathrm{l}$ of the Halt Protease Inhibitor Single-Use Cocktail (Thermo Scientific) was added. Cell disruption was performed through 3 cycles of freezing (liquid nitrogen) and thawing, followed by re-solubilization in $7 \mathrm{M}$ urea, $2 \mathrm{M}$ thiourea, 1\% DTT and 4\% CHAPS containing 0.5\% ampholytes, pH 5-8 (Bio-Rad, Cressier, Switzerland), operating a Bioruptor ${ }^{\circ}$ UCD-200 for $15 \mathrm{~min}$ at high intensity. Twenty-five micrograms of protein (determined by the Bradford Assay) was applied to an IEF strip (Bio-Rad) via in-gel re-hydration for $12 \mathrm{~h}$ at $50 \mathrm{mV}$, after which isoelectric focusing (IEF) was performed for a total of 32 $\mathrm{kVh}$. After IEF, the strips were reduced in equilibration buffer (6 M urea, $50 \mathrm{mM}$ Tris pH 8.8, 2\% SDS, 30\% glycerol) containing 1\% DTT for $10 \mathrm{~min}$, followed by alkylation in equilibration buffer containing $4 \%$ iodoacetamide for $10 \mathrm{~min}$. The second dimension was run on a precast 4-15\% gradient polyacrylamide gel (Bio-Rad) at a constant voltage of $200 \mathrm{~V}$. The separated proteins were visualized using the SilverQuest ${ }^{\text {ti }}$ Silver Staining Kit (Invitrogen) according to the manufacturer's protocol. Protein spots to be analyzed by nano-LC MS/MS were excised and destained with $50 \mu \mathrm{l}$ of Destainer A and $50 \mu \mathrm{l}$ of Destainer B (SilverQuest ${ }^{\text {tux }}$ Silver Staining Kit). For each condition, spots from three 2D gels were analyzed.

\section{D gel electrophoresis}

To obtain an additional, broader overview of the N. fowleri proteome, proteins from weakly as well as highly pathogenic trophozoites were separated by 1D gel electrophoresis and identified through nano-LC MS/MS. Pellets of $10^{7}$ trophozoites were resolubilized for $3 \mathrm{~min}$ via sonication in a water bath with $5 \mathrm{mM}$ HEPES, $\mathrm{pH}$ 7.4, and $50 \mathrm{mM}$ mannitol containing a protease inhibitor cocktail (Roche). Protein concentrations were determined by OD280 $\mathrm{nm}$ measurement, and aliquots 
corresponding to $30 \mu \mathrm{g}$ of protein were separated by SDS-PAGE (10\%). Each sample lane was cut into 27 slices from top to bottom for in-gel digestion and nano-LC MS/MS.

\section{Nano-LC MS/MS}

For protein identification, protein spots excised following 2D gel electrophoresis and slices excised following 1D gel electrophoresis were further processed for nanoLC MS/MS analysis. The 2D gel spots were digested directly, while the gel slices were reduced and alkylated prior to digestion with trypsin and analyzed by nano-LC MS/MS as described in [70]. The generated fragment spectra were searched against our personal ORF database obtained from RNA sequencing (see above) using EasyProt software [71].

\section{Data mining and annotation}

Based on data from 1D gel electrophoresis, in combination with nano-LC MS/MS, differentially expressed proteins were identified by summing all of the scores from peptide spectral matches to one particular ORF, which is termed protein match score summation (PMSS) [72], and calculation of the relationship of the PMSS of highly pathogenic to weakly pathogenic $N$. fowleri. Then, ORFs with a relative PMSS value equal to or greater than 2, or equal to or less than -2 were considered to be differentially expressed proteins between the two conditions.

For annotation, the differentially expressed ORFs were subjected to searches with the next-generation sequence similarity search tool ngKLAST (www.korilog.com). A KLASTp search was run against the annotated Swissprot database under default settings. KLAST hits with a bit score greater than or equal to 50 were considered significant and were used for further analysis.

Data clustering based on GO terms was carried out on the $\mathrm{R}$ platform for statistical programming using packages from the Bioconductor project [73]. To retrieve GO identifiers associated with the Uniprot Accession numbers of significant KLAST hits, the biomaRt package was used, which implements the BioMart software suite $[74,75]$.

\section{Additional file}

Additional file 1: Primer sequences. Primers used to determine the contents of ribosomal and mitochondrial DNA relative to the genomic DNA used as the starting material for genome sequencing.

\section{Abbreviations}

AIP1: Apoptosis-linked gene-2-interacting protein X1; CLC: CLC Genomic Workbench; EGN: Evolutionary Gene and Genome Network; GO: Gene Ontology; Hsp70: Heat shock protein 70; IEF: Isoelectric focussing; LH: Liver hydrolysate; Nano-LC MS/MS: Nano-liquid chromatography tandem mass spectrometry; NCBI: National Center for Biotechnology Information; ORFs: Open reading frames; PAM: Primary amoebic meningoencephalitis;
PMSS: Protein match score summation; RPGR: Retinitis pigmentosa GTPase regulator.

\section{Competing interests}

The authors declared that they have no competing interest.

\section{Authors' contribution}

MW and NM conceived and designed the study. DCZ and MW wrote the manuscript. DCZ and MH performed the experiments. DCZ, MW and CB analyzed and interpreted the data. BG and NS contributed their expertise in the field of parasitology. All authors read and approved the final manuscript.

\section{Acknowledgements}

We would like to thank the Next Generation Sequencing Platform of the University of Bern for performing the high-throughput sequencing experiments. Furthermore, we thank Vidhya Jagannathan for her support in the processing of the RNA sequencing data, Caroline Frey for kindly providing Trichomonas vaginalis and Dorothea Nillius for the Giardia lamblia cells. The study was funded by the Federal Office for Civil Protection (project number 353002433).

\section{Author details}

${ }^{1}$ Biology Division, Spiez Laboratory, Federal Office for Civil Protection, Austrasse, CH-3700 Spiez, Switzerland. ${ }^{2}$ Institute of Parasitology, University of Bern, Länggassstrasse 122, CH-3012 Bern, Switzerland. ${ }^{3}$ Graduate School for Cellular and Biomedical Sciences, University of Bern, Freiestrasse 1, CH-3012 Bern, Switzerland. ${ }^{4}$ Mass Spectrometry and Proteomics, Department of Clinical Research, University Hospital, Bern CH-3010, Switzerland.

Received: 4 December 2013 Accepted: 11 June 2014

Published: 19 June 2014

\section{References}

1. De Jonckheere J, Voorde $\mathrm{H}$ : The distribution of Naegleria fowleri in man-made thermal waters. Am J Trop Med Hyg 1977, 26:10-15.

2. De Jonckheere JF: Molecular definition and the ubiquity of species in the genus Naegleria. Protist 2004, 155:89-103.

3. Carter RF: Description of a Naegleria sp. isolated from two cases of primary amoebic meningo-encephalitis, and of the experimental pathological changes induced by it. J Pathol 1970, 100:217-244.

4. Jarolim KL, McCosh JK, Howard MJ, John DT: A light microscopy study of the migration of Naegleria fowleri from the nasal submucosa to the central nervous system during the early stage of primary amebic meningoencephalitis in mice. J Parasitol 2000, 86:50-55.

5. Schuster FL, Visvesvara GS: Free-living amoebae as opportunistic and non-opportunistic pathogens of humans and animals. Int J Parasitol 2004, 34:1001-1027.

6. Debnath A, Tunac JB, Galindo-Gomez S, Silva-Olivares A, Shibayama M, McKerrow JH: Corifungin, a new drug lead against Naegleria, identified from a high-throughput screen. Antimicrob Agents Chemother 2012 56:5450-5457.

7. Vargas-Zepeda J, Gomez-Alcala AV, Vasquez-Morales JA, Licea-Amaya L, De Jonckheere JF, Lares-Villa F: Successful treatment of Naegleria fowleri meningoencephalitis by using intravenous amphotericin B, fluconazole and rifampicin. Arch Med Res 2005, 36:83-86.

8. Fritz-Laylin LK, Prochnik SE, Ginger ML, Dacks JB, Carpenter ML, Field MC, Kuo A, Paredez A, Chapman J, Pham J, Shu S, Neupane R, Cipriano M, Mancuso J, Tu H, Salamov A, Lindquist E, Shapiro H, Lucas S, Grigoriev IV, Cande WZ, Fulton C, Rokhsar DS, Dawson SC: The genome of Naegleria gruberi illuminates early eukaryotic versatility. Cell 2010, 140:631-642.

9. Herman EK, Greninger AL, Visvesvara GS, Marciano-Cabral F, Dacks JB, Chiu CY: The mitochondrial genome and a 60-kb nuclear DNA segment from Naegleria fowleri, the causative agent of primary amoebic meningoencephalitis. J Eukaryot Microbiol 2013, 60:179-191.

10. Burri DC, Gottstein B, Zumkehr B, Hemphill A, Schürch N, Wittwer M, Müller N: Development of a high- versus low-pathogenicity model of the free-living amoeba Naegleria fowleri. Microbiology 2012, 158:2652-2660.

11. Jung SY, Kim JH, Lee YJ, Song KJ, Kim K, Park S, Im KI, Shin HJ: Naegleria fowleri: nfa1 gene knock-down by double-stranded RNAs. Exp Parasitol 2008, 118:208-213. 
12. Sohn HJ, Kim JH, Shin MH, Song KJ, Shin HJ: The Nf-actin gene is an important factor for food-cup formation and cytotoxicity of pathogenic Naegleria fowleri. Parasitol Res 2010, 106:917-924.

13. Jung SY, Kim JH, Song KJ, Lee YJ, Kwon MH, Kim K, Park S, Im Kl, Shin HJ: Gene silencing of $n f a 1$ affects the in vitro cytotoxicity of Naegleria fowleri in murine macrophages. Mol Biochem Parasitol 2009, 165:87-93.

14. Lee YJ, Kim JH, Jeong SR, Song KJ, Kim K, Park S, Park MS, Shin HJ: Production of $\mathrm{Nfa} 1$-specific monoclonal antibodies that influences the in vitro cytotoxicity of Naegleria fowleri trophozoites on microglial cells. Parasitol Res 2007, 101:1191-1196.

15. Jeong SR, Lee SC, Song KJ, Park S, Kim K, Kwon MH, Im KI, Shin HJ: Expression of the nfal gene cloned from pathogenic Naegleria fowleri in nonpathogenic $N$. gruberi enhances cytotoxicity against $\mathrm{CHO}$ target cells in vitro. Infect Immun 2005, 73:4098-4105.

16. Jeong SR, Kang SY, Lee SC, Song KJ, Im Kl, Shin HJ: Decreasing effect of an anti-Nfa1 polyclonal antibody on the in vitro cytotoxicity of pathogenic Naegleria fowleri. Korean J Parasitol 2004, 42:35-40.

17. Oh YH, Jeong SR, Kim JH, Song KJ, Kim K, Park S, Sohn S, Shin HJ: Cytopathic changes and pro-inflammatory cytokines induced by Naegleria fowleri trophozoites in rat microglial cells and protective effects of an anti-Nfa1 antibody. Parasite Immunol 2005, 27:453-459.

18. Kang SY, Song KJ, Jeong SR, Kim JH, Park S, Kim K, Kwon MH, Shin HJ: Role of the Nfa1 protein in pathogenic Naegleria fowleri cocultured with $\mathrm{CHO}$ target cells. Clin Diagn Lab Immunol 2005, 12:873-876.

19. Reveiller FL, Suh SJ, Sullivan K, Cabanes PA, Marciano-Cabral F: Isolation of a unique membrane protein from Naegleria fowleri. J Eukaryot Microbiol 2001, 48:676-682.

20. Baverstock PR, Illana S, Christy PE, Robinson BS, Johnson AM: srRNA evolution and phylogenetic relationships of the genus Naegleria (Protista: Rhizopoda). Mol Biol Evol 1989, 6:243-257.

21. De Jonckheere JF: Origin and evolution of the worldwide distributed pathogenic amoeboflagellate Naegleria fowleri. Infect Genet Evol 2011, 11:1520-1528

22. Marciano-Cabral F, Cabral GA: The immune response to Naegleria fowleri amebae and pathogenesis of infection. FEMS Immunol Med Microbiol 2007, 51:243-259.

23. Han KL, Lee HJ, Shin MH, Shin HJ, Im Kl, Park SJ: The involvement of an integrin-like protein and protein kinase $\mathrm{C}$ in amoebic adhesion to fibronectin and amoebic cytotoxicity. Parasitol Res 2004, 94:53-60.

24. Brown T: Observations by light microscopy on the cytopathogenicity of Naegleria fowleri in mouse embryo-cell cultures. J Med Microbiol 1978, 11:249-259.

25. Marciano-Cabral FM, Patterson M, John DT, Bradley SG: Cytopathogenicity of Naegleria fowleri and Naegleria gruberi for established mammalian cell cultures. J Parasitol 1982, 68:1110-1116.

26. Marciano-Cabral FM, Fulford DE: Cytopathology of pathogenic and nonpathogenic Naegleria species for cultured rat neuroblastoma cells. Appl Environ Microbiol 1986, 51:1133-1137.

27. Lowrey DM, McLaughlin J: Activation of a heat-stable cytolytic protein associated with the surface membrane of Naegleria fowleri. Infect Immun 1985, 50:478-482.

28. Toney DM, Marciano-Cabral F: Alterations in protein expression and complement resistance of pathogenic Naegleria amoebae. Infect Immun 1992, 60:2784-2790.

29. Fritzinger AE, Toney DM, MacLean RC, Marciano-Cabral F: Identification of a Naegleria fowleri membrane protein reactive with anti-human CD59 antibody. Infect Immun 2006, 74:1189-1195.

30. Toney DM, Marciano-Cabral F: Membrane vesiculation of Naegleria fowleri amoebae as a mechanism for resisting complement damage. J Immunol 1994, 152:2952-2959.

31. Herbst R, Ott C, Jacobs T, Marti T, Marciano-Cabral F, Leippe M: Pore-forming polypeptides of the pathogenic protozoon Naegleria fowleri. J Biol Chem 2002, 277:22353-22360

32. Matsuo H, Chevallier J, Mayran N, Le Blanc I, Ferguson C, Faure J, Blanc NS, Matile S, Dubochet J, Sadoul R, Parton RG, Vilbois F, Gruenberg J: Role of LBPA and Alix in multivesicular liposome formation and endosome organization. Science 2004, 303:531-534.

33. Wang $L$, Zhan $Y$, Song E, Yu Y, Jiu Y, Du W, Lu J, Liu P, Xu P, Xu T: HID-1 is a peripheral membrane protein primarily associated with the medial- and trans- Golgi apparatus. Protein Cell 2011, 2:74-85.

34. Yu Y, Wang L, Jiu Y, Zhan Y, Liu L, Xia Z, Song E, Xu P, Xu T: HID-1 is a novel player in the regulation of neuropeptide sorting. Biochem J 2011, 434:383-390
35. Lynch EC, Rosenberg IM, Gitler C: An ion-channel forming protein produced by Entamoeba histolytica. EMBO J 1982, 1:801-804.

36. Que X, Reed SL: Cysteine proteinases and the pathogenesis of amebiasis. Clin Microbiol Rev 2000, 13:196-206.

37. Juarez-Hernandez LJ, Garcia-Perez RM, Salas-Casas A, Garcia-Rivera G, Orozco E, Rodriguez MA: Entamoeba histolytica: the over expression of a mutated EhRabB protein produces a decrease of in vitro and in vivo virulence. Exp Parasitol 2013, 133:339-345.

38. Hernandes-Alejandro M, Calixto-Galvez M, Lopez-Reyes I, Salas-Casas A, Cazares-Apatiga J, Orozco E, Rodriguez MA: The small GTPase EhRabB of Entamoeba histolytica is differentially expressed during phagocytosis. Parasitol Res 2013, 112:1631-1640.

39. Bosch DE, Siderovski DP: G protein signaling in the parasite Entamoeba histolytica. Exp Mol Med 2013, 45:e15.

40. Brown T: Observations by immunofluorescence microscopy and electron microscopy on the cytopathogenicity of Naegleria fowleri in mouse embryo-cell cultures. J Med Microbiol 1979, 12:363-371.

41. Marciano-Cabral F, John DT: Cytopathogenicity of Naegleria fowleri for rat neuroblastoma cell cultures: scanning electron microscopy study. Infect Immun 1983, 40:1214-1217.

42. Kim JH, Lee SH, Sohn HJ, Lee J, Chwae YJ, Park S, Kim K, Shin HJ: The immune response induced by DNA vaccine expressing $n f a 1$ gene against Naegleria fowleri. Parasitol Res 2012, 111:2377-2384.

43. Kim JH, Sohn HJ, Lee J, Yang HJ, Chwae YJ, Kim K, Park S, Shin HJ: Vaccination with lentiviral vector expressing the $n f a 1$ gene confers a protective immune response to mice infected with Naegleria fowleri. Clin Vaccine Immunol 2013, 20:1055-1060.

44. Song KJ, Song KH, Kim JH, Sohn HJ, Lee YJ, Park CE, Shin HJ: Heat shock protein 70 of Naegleria fowleri is important factor for proliferation and in vitro cytotoxicity. Parasitol Res 2008, 103:313-317.

45. Gumbiner BM: Cell adhesion: the molecular basis of tissue architecture and morphogenesis. Cell 1996, 84:345-357.

46. Bretscher A: Microfilament structure and function in the cortical cytoskeleton. Annu Rev Cell Biol 1991, 7:337-374.

47. Voigt $H$, Guillen $N$ : New insights into the role of the cytoskeleton in phagocytosis of Entamoeba histolytica. Cell Microbiol 1999, 1:195-203.

48. Gonzalez-Robles A, Castanon G, Hernandez-Ramirez VI, Salazar-Villatoro L, Gonzalez-Lazaro M, Omana-Molina M, Talamas-Rohana P, Martinez-Palomo A: Acanthamoeba castellanii: identification and distribution of actin cytoskeleton. Exp Parasitol 2008, 119:411-417.

49. Chesarone MA, DuPage AG, Goode BL: Unleashing formins to remodel the actin and microtubule cytoskeletons. Nat Rev Mol Cell Biol 2010, $11: 62-74$

50. Higgs HN: Formin proteins: a domain-based approach. Trends Biochem Sci 2005, 30:342-353.

51. Matsudaira P, Janmey P: Pieces in the actin-severing protein puzzle. Cell 1988, 54:139-140.

52. Pavlov D, Muhlrad A, Cooper J, Wear M, Reisler E: Actin filament severing by cofilin. J Mol Biol 2007, 365:1350-1358.

53. Bamburg JR: Proteins of the ADF/cofilin family: essential regulators of actin dynamics. Annu Rev Cell Dev Biol 1999, 15:185-230.

54. Khurana S, George SP: Regulation of cell structure and function by actin-binding proteins: villin's perspective. FEBS Lett 2008, 582:2128-2139.

55. Gakovic M, Shu X, Kasioulis I, Carpanini S, Moraga I, Wright AF: The role of RPGR in cilia formation and actin stability. Hum Mol Genet 2011, 20:4840-4850.

56. Diakonova M, Bokoch G, Swanson JA: Dynamics of cytoskeletal proteins during Fcgamma receptor-mediated phagocytosis in macrophages. $\mathrm{Mol}$ Biol Cell 2002, 13:402-411.

57. Araki N, Hatae T, Furukawa A, Swanson JA: Phosphoinositide-3-kinaseindependent contractile activities associated with Fcgamma-receptormediated phagocytosis and macropinocytosis in macrophages. J Cell Sci 2003, 116:247-257

58. Coluccio LM: Myosin I. Am J Physiol 1997, 273:C347-C359.

59. De Jonckheere JF: Variation of electrophoretic karyotypes among Naegleria spp. Parasitol Res 1989, 76:55-62.

60. Clark CG, Cross GA, De Jonckheere JF: Evaluation of evolutionary divergence in the genus Naegleria by analysis of ribosomal DNA plasmid restriction patterns. Mol Biochem Parasitol 1989, 34:281-296.

61. Dolezel J, Bartos J: Plant DNA flow cytometry and estimation of nuclear genome size. Ann Bot 2005, 95:99-110. 
62. Ronildo Clarindo W, Roberto Carvalho C: Flow cytometric analysis using SYBR Green I for genome size estimation in coffee. Acta Histochem 2011, 113:221-225.

63. McArthur AG, Morrison HG, Nixon JE, Passamaneck NQ, Kim U, Hinkle G, Crocker MK, Holder ME, Farr R, Reich Cl, Olsen GE, Aley SB, Adam RD, Gillin FD, Sogin ML: The Giardia genome project database. FEMS Microbiol Lett 2000, 189:271-273.

64. Zubacova Z, Cimburek Z, Tachezy J: Comparative analysis of trichomonad genome sizes and karyotypes. Mol Biochem Parasitol 2008, 161:49-54.

65. Hare EE, Johnston JS: Genome size determination using flow cytometry of propidium iodide-stained nuclei. Methods Mol Biol 2011, 772:3-12.

66. Grabherr MG, Haas BJ, Yassour M, Levin JZ, Thompson DA, Amit I, Adiconis X, Fan L, Raychowdhury R, Zeng Q, Chen Z, Mauceli E, Hacohen N, Gnirke A, Rhind N, di Palma F, Birren BW, Nusbaum C, Lindblad-Toh K, Friedman N, Regev A: Full-length transcriptome assembly from RNA-Seq data without a reference genome. Nat Biotechnol 2011, 29:644-652.

67. Halary S, Mclnerney JO, Lopez P, Bapteste E: EGN: a wizard for construction of gene and genome similarity networks. BMC Evol Biol 2013, 13:146.

68. Cline MS, Smoot M, Cerami E, Kuchinsky A, Landys N, Workman C, Christmas R, Avila-Campilo I, Creech M, Gross B, Hanspers K, Isserlin R, Kelley R, Killcoyne S, Lotia S, Maere S, Morris J, Ono K, Pavlovic V, Pico AR, Vailaya A, Wang PL, Adler A, Conklin BR, Hood L, Kuiper M, Sander C, Schmulevich I, Schwikowski B, Warner GJ, et al: Integration of biological networks and gene expression data using Cytoscape. Nat Protoc 2007, 2:2366-2382.

69. Altschul SF, Gish W, Miller W, Myers EW, Lipman DJ: Basic local alignment search tool. J Mol Biol 1990, 215:403-410.

70. Al Kaabi A, Traupe T, Stutz M, Buchs N, Heller M: Cause or effect of arteriogenesis: compositional alterations of microparticles from CAD patients undergoing external counterpulsation therapy. PLoS One 2012, 7:e46822.

71. Gluck F, Hoogland C, Antinori P, Robin X, Nikitin F, Zufferey A, Pasquarello C, Fetaud V, Dayon L, Müller M, Lisacek F, Geiser L, Hochstrasser D, Sanchez JC, Scherl A: EasyProt-an easy-to-use graphical platform for proteomics data analysis. J Proteomics 2013, 79:146-160.

72. Heller M, Schlappritzi E, Stalder D, Nuoffer JM, Haeberli A: Compositional protein analysis of high density lipoproteins in hypercholesterolemia by shotgun LC-MS/MS and probabilistic peptide scoring. Mol Cell Proteomics 2007, 6:1059-1072.

73. Gentleman RC, Carey VJ, Bates DM, Bolstad B, Dettling M, Dudoit S, Ellis B, Gautier L, Ge Y, Gentry J, Hornik K, Hothorn T, Huber W, lacus S, Irizarry R, Leisch F, Li C, Maechler M, Rossini AJ, Sawitzki G, Smith C, Smyth G, Tierney L, Yang JY, Zhang J: Bioconductor: open software development for computational biology and bioinformatics. Genome Biol 2004, 5:R80.

74. Kasprzyk A: BioMart: driving a paradigm change in biological data management. Database (Oxford) 2011, 2011:bar049.

75. Zhang J, Haider S, Baran J, Cros A, Guberman JM, Hsu J, Liang Y, Yao L, Kasprzyk A: BioMart: a data federation framework for large collaborative projects. Database (Oxford) 2011, 2011:bar038.

doi:10.1186/1471-2164-15-496

Cite this article as: Zysset-Burri et al: Genome-wide identification of pathogenicity factors of the free-living amoeba Naegleria fowleri. BMC Genomics 2014 15:496.

\section{Submit your next manuscript to BioMed Central and take full advantage of:}

- Convenient online submission

- Thorough peer review

- No space constraints or color figure charges

- Immediate publication on acceptance

- Inclusion in PubMed, CAS, Scopus and Google Scholar

- Research which is freely available for redistribution

Submit your manuscript at www.biomedcentral.com/submit 